\title{
Dilute solution properties of poly(di-tert-butyl fumarate)
}

\author{
Nozomi Awazu, Takuya Komatsubara, Masashi Osa ${ }^{1}$, Takenao Yoshizaki and Jiro Shimada
}

The mean-square radius of gyration $\left\langle S^{2}\right\rangle$ and second virial coefficient $A_{2}$ are determined from static light scattering (LS) measurements for six samples of poly(di-tert-butyl fumarate) (PDtBF) for a range of weight-average molecular weight $M_{\mathrm{w}}$ from $4.35 \times 10^{4}$ to $1.73 \times 10^{5}$ in tetrahydrofuran at $30.0^{\circ} \mathrm{C}$. The intrinsic viscosity $[\eta]$ and translational diffusion coefficient $D$ are also determined from viscosity and dynamic LS measurements for those samples under the same solvent conditions. From a simultaneous analysis of $\left\langle S^{2}\right\rangle$ and $A_{2}$ based on the Kratky-Porod (KP) wormlike chain with excluded volume, the stiffness parameter $\lambda^{-1}$ and the reduced excluded-volume strength $\lambda B$ are determined to be $350 \AA$ and 0.024 , respectively, where we assume the shift factor $M_{\mathrm{L}}$ for PDtBF to be $101 \AA^{-1}$ on the analogy of the main-chain structure of poly(diisopropyl fumarate) previously studied. For PDtBF with such a large $\lambda^{-1}$, it is found that the intramolecular excluded-volume effect for $\left\langle S^{2}\right\rangle$ is negligibly small in the range of $M_{\mathrm{w}}$ examined. As for $[\eta]$ and $D$, their behavior may be well explained by the corresponding KP theories using the model parameter values consistent with those determined from $\left\langle S^{2}\right\rangle$ and $A_{2}$, and the intramolecular excludedvolume effect for them can be ignored, as in the case of $\left\langle S^{2}\right\rangle$.

Polymer Journal (2016) 48, 991-997; doi:10.1038/pj.2016.63; published online 29 June 2016

\section{INTRODUCTION}

Recently, we conducted a study of the dilute solution properties of poly(diisopropyl fumarate) (PDiPF), which has ester isopropyl groups on every main-chain carbon atom, by determining its mean-square radius of gyration $\left\langle S^{2}\right\rangle$, second virial coefficient $A_{2}$, intrinsic viscosity $[\eta]$ and translational diffusion coefficient $D$ in tetrahydrofuran (THF) at $30.0{ }^{\circ} \mathrm{C}$ (good solvent). ${ }^{1,2}$ Because of the steric hindrance of the bulky substituents preventing internal rotations around the mainchain $\mathrm{C}-\mathrm{C}$ bonds, the PDiPF chain was predicted to be stiffer than common vinyl polymer chains having substituents on every other main-chain carbon atom. ${ }^{3-5}$ From a simultaneous analysis of the experimental results for $\left\langle S^{2}\right\rangle$ and $A_{2}$ based on the Kratky-Porod (KP) wormlike chain model ${ }^{6,7}$ with a consideration of the intramolecular and intermolecular excluded-volume effects, the values of the stiffness parameter $\lambda^{-1}$, the shift factor $M_{\mathrm{L}}$ as defined as the molecular weight per unit contour length of the KP chain and the reduced excludedvolume strength ${ }^{7} \lambda B$ have been determined to be $113 \AA, 89 \AA^{-1}$ and 0.097 , respectively. ${ }^{1}$ It has then been concluded from the value of $\lambda^{-1}$ so determined that the PDiPF chain is stiffer than typical flexible polymers, such as atactic polystyrene $\left(\lambda^{-1}=20.6 \AA\right), 7,8$ as was expected. For $[\eta]$ and $D$ (or the hydrodynamic radius $R_{\mathrm{H}}$ ) of PDiPF, it has been shown that the experimental values agree well with the corresponding perturbed KP theory ones using the model parameter values consistent with the above ones determined from $\left\langle S^{2}\right\rangle$ and $A_{2}{ }^{2}$

Poly(di-tert-butyl fumarate) (PDtBF) with ester tert-butyl groups on every main-chain carbon atom belongs to the same category as PDiPF. Because the ester tert-butyl group of PDtBF is bulkier than the ester isopropyl group of PDiPF, the hindrance to the internal rotations is considered to be greater in the PDtBF chain than in the PDiPF one. Therefore, the former is expected to be still stiffer than the latter. In order to confirm this expectation, in the present study, we make a study of the dilute solution properties of PDtBF. We determine $\left\langle S^{2}\right\rangle$, $A_{2},[\eta]$ and $D$ for PDtBF under the same solvent condition as in the case of PDiPF and evaluate $\lambda^{-1}$ for PDtBF from analyses of the experimental data based on the KP model.

The analyses of $\left\langle S^{2}\right\rangle,[\eta]$ and $R_{\mathrm{H}}$ of a semiflexible polymer in a good solvent require a comment. If its $\lambda^{-1}$ is large and its weight-average molecular weight $M_{\mathrm{w}}$ is not very high, as in the case of typical semiflexible polymers with large $\lambda^{-1}$ such as $\operatorname{poly}(n$-hexyl isocyanate) with $\lambda^{-1}=840 \AA$, ${ }^{9}$ then the intramolecular excluded-volume effect on those quantities may be negligibly small. In the previous case of PDiPF, ${ }^{1,2}$ however, the value $113 \AA$ of $\lambda^{-1}$ is not large enough to ignore the effect in the range of $M_{\mathrm{w}}$ examined. Because there is currently no available information regarding the stiffness of the PDtBF chain, the analyses of $\left\langle S^{2}\right\rangle,[\eta]$ and $R_{\mathrm{H}}$ of PDtBF should be carried out with the consideration of possible intramolecular excluded-volume effects. Therefore, we first analyze $\left\langle S^{2}\right\rangle$ and $A_{2}$ of PDtBF simultaneously on the basis of the quasi-two-parameter (QTP) scheme $e^{7,10-12}$ for the intramolecular excluded-volume effect and also the Yamakawa theory ${ }^{7,13}$ of $A_{2}$ for the intermolecular excluded-volume effect. From the analysis, we determine the values of $\lambda^{-1}$ and $\lambda B$ for PDtBF, with the value of $M_{\mathrm{L}}$ for PDtBF estimated by analogy with that for PDiPF. Then we compare the experimental data for $[\eta]$ and $D$ with the corresponding KP theories combined with the QTP scheme, to ascertain the validity of the model parameter values determined from $\left\langle S^{2}\right\rangle$ and $A_{2}$. 


\section{EXPERIMENTAL PROCEDURE}

\section{Materials}

The original PDtBF samples were synthesized by the radical polymerization of di-tert-butyl fumarate (DtBF), according to the procedure described by Otsu et al. ${ }^{14}$ and Crivello and Shim. ${ }^{15}$ Polymerization was carried out in benzene using dimethyl 2,2'-azobis(isobutyrate) (Wako Pure Chemical Industries, Ltd, Osaka, Japan) as an initiator under dry nitrogen at $60^{\circ} \mathrm{C}$ for $24 \mathrm{~h}$. The monomer DtBF was synthesized by the reaction of fumaryl chloride (Wako Pure Chemical Industries) with potassium tert-butoxide (Wako Pure Chemical Industries) in tert-butanol at reflux for $4 \mathrm{~h}$. The synthesized PDtBF samples were purified by reprecipitation from benzene solutions into methanol and subsequently separated into fractions with narrow molecular weight distribution by fractional precipitation using toluene as a solvent and methanol as a precipitant. The six prepared test samples were freeze-dried from their benzene solutions.

The ratios of $M_{\mathrm{w}}$ to the number-average molecular weight $M_{\mathrm{n}}$ for all of the samples were determined from analytical gel permeation chromatography using THF as an eluent and standard polystyrene samples (Tosoh Corporation, Tokyo, Japan) as reference standards.

The THF solvent used for static light scattering (SLS), dynamic light scattering (DLS) and viscosity measurements was purified by distillation after refluxing over sodium. The THF solvent used for analytical gel permeation chromatography was of reagent grade.

\section{Static light scattering}

SLS measurements were carried out to determine $M_{\mathrm{w}}$ and $A_{2}$ for the six test samples and $\left\langle S^{2}\right\rangle$ for four test samples with $M_{\mathrm{w}}>7 \times 10^{4}$, in THF at $30.0^{\circ} \mathrm{C}$. The apparatus employed for the SLS measurements was the same as that used in the previous study for PDiPF. ${ }^{1}$ The wavelength of the incident light was $436 \mathrm{~nm}$. For each sample, at six different concentrations and at scattering angles $\theta$ ranging from 30.0 to $142.5^{\circ}$, the scattered light intensity was measured. We treated the obtained data by using the Berry square-root plot. ${ }^{16}$ For all of the samples, the degree of depolarization was negligibly small, so that corrections for the optical anisotropy were unnecessary.

The most concentrated solution of each sample was prepared gravimetrically and then stirred continuously in the dark at room temperature for 2 days, to ensure homogeneity. For optical purification, the solution was filtered through a Teflon membrane Fluoropore (Sumitomo Electric Industries, Ltd, Osaka, Japan) with a pore size of $0.10 \mu \mathrm{m}$. To obtain the solutions of lower concentrations, the most concentrated solution was successively diluted. The polymer mass concentrations $c$ were converted from the weight concentrations by using the densities of each solution, which were calculated with the partial specific volumes $v_{2}$ of the samples and with the density $\rho_{0}$ of the solvent THF. $v_{2}$ and $\rho_{0}$ were measured using an oscillating U-tube density meter DMA5000 (Anton-Paar, Graz, Austria). For all of the samples, the values of $v_{2}$ in THF at $30.0^{\circ} \mathrm{C}$ are $0.876 \mathrm{~cm}^{3} \mathrm{~g}^{-1}$. The value of $\rho_{0}$ for THF at $30.0^{\circ} \mathrm{C}$ is $0.8751 \mathrm{~g} \mathrm{~cm}^{-3}$.

The measurements of the refractive index increment $\partial n / \partial c$ were carried out by using the same apparatus as in the previous study for PDiPF. ${ }^{1}$ For all of the samples, the values of $\partial n / \partial c$ in THF at $30.0^{\circ} \mathrm{C}$ and at the wavelength of $436 \mathrm{~nm}$ are $0.0770 \mathrm{~cm}^{3} \mathrm{~g}^{-1}$. The refractive index $n_{0}$ of THF at $30.0^{\circ} \mathrm{C}$ and at the wavelength of $436 \mathrm{~nm}$ is $1.4108 .{ }^{1}$

\section{Viscosity}

Viscosity measurements were carried out for the six samples in THF at $30.0^{\circ} \mathrm{C}$, using a conventional capillary viscometer of the Ubbelohde type, to determine $[\eta]$ and the Huggins coefficient $k^{\prime}$ of the samples. The details of the procedures of the measurements, data collection and data processing have been described in a previous study. ${ }^{2}$

\section{Dynamic light scattering}

DLS measurements were carried out to determine $D$ for all of the samples except PDtBF4 in THF at $30.0^{\circ} \mathrm{C}$. The apparatus employed for the DLS measurements was the same as that used in the previous study for PDiPF. ${ }^{2}$ The wavelength of the incident light was $532 \mathrm{~nm}$. For each sample, at 3-6 concentrations and at scattering angles $\theta$ ranging from 30 to $50^{\circ}$, the normalized autocorrelation function $g^{(2)}(t)$ of the scattered light intensity was measured. The preparation and the optical purification of the solutions were made in the same manner as in the cases of the SLS measurements. The details of the data acquisition and data processing have been described in previous studies. ${ }^{2,17,18}$ The values of $n_{0}$ at the wavelength of $532 \mathrm{~nm}$ and of the viscosity coefficient $\eta_{0}$ for THF at $30.0^{\circ} \mathrm{C}$ are 1.4045 and $0.439_{7} \mathrm{cP}$, respectively. ${ }^{2}$

\section{RESULTS}

The values of $M_{\mathrm{w}}$ determined from SLS measurements in THF at $30.0^{\circ} \mathrm{C}$ and $M_{\mathrm{w}} / M_{\mathrm{n}}$ from analytical gel permeation chromatography for all the six PDtBF samples are listed in the second and third columns of Table 1, respectively. In the fourth column of Table 1 are listed the values of the weight-average number of repeat units $n_{\mathrm{w}}$ calculated by the following equation,

$$
n_{\mathrm{w}}=2 M_{\mathrm{w}} / M_{0}
$$

where $M_{0}$ is the molecular weight of the monomer unit. We note that the values of $M_{0}$ are 228 and 200 for PDtBF and PDiPF, respectively. We also note that the range of $M_{\mathrm{w}}$ (and also $n_{\mathrm{w}}$ ) for PDtBF is somewhat narrower than that for the PDiPF previously investigated, ${ }^{1,2}$ probably because the applied polymerization time for the former samples was shorter than that for the latter ones.

As for the stereochemical compositions of PDtBF prepared by radical polymerization, Yoshioka et al. ${ }^{19,20}$ have reported that the fraction $f_{\mathrm{r}}$ of racemo diads depends on the polymerization temperature and that the value of $f_{\mathrm{r}}$ for the PDtBF samples synthesized at $60^{\circ} \mathrm{C}$ is 0.14. Therefore, we think that the values of $f_{\mathrm{r}}$ for the present PDtBF samples are approximately 0.14 . This value is not far from the $f_{\mathrm{r}}$ value 0.22 for the PDiPF samples that have previously ${ }^{1,2}$ been studied. Here it should be noted that if vicinal substituents joined to a pair of adjacent backbone carbon atoms (diad) in a PDtBF (or PDiPF) chain are located on opposite sides of a plane containing all of the backbone carbon atoms of the chain in the planer trans conformation, then the diad is called meso; otherwise, the diad is called racemo. ${ }^{21}$

\section{Mean-square radius of gyration $\left\langle S^{2}\right\rangle$}

The values of $\left\langle S^{2}\right\rangle$ determined from the SLS measurements for the PDtBF samples in THF at $30.0^{\circ} \mathrm{C}$ are listed in the fifth column of Table 1. In the table, the values of $\left\langle S^{2}\right\rangle$ for the samples PDtBF4 and PDtBF5 have been excluded because the values of $\left\langle S^{2}\right\rangle$ for these samples are too small to determine accurately by the SLS measurements.

Figure 1 shows the double-logarithmic plots of the ratio of $\left\langle S^{2}\right\rangle$ $\left(\left\langle S^{2}\right\rangle\right.$ in $\left.\AA^{2}\right)$ to $n_{\mathrm{w}}$ against $n_{\mathrm{w}}$. The unfilled circles represent the present experimental data for PDtBF in THF at $30.0^{\circ} \mathrm{C}$. The solid and dashed curves represent the theoretical values for the KP chain with and without the excluded volume, respectively, which are discussed in the Discussion section. $\left\langle S^{2}\right\rangle / n_{\mathrm{w}}$ for PDtBF increases monotonically with increasing $n_{\mathrm{w}}$.

Table 1 Values of $M_{\mathrm{w}} / M_{\mathrm{n}}$ and $n_{\mathrm{w}}$ and the results of the SLS measurements for poly(di-tert-butyl fumarate) in tetrahydrofuran at $30.0^{\circ} \mathrm{C}$

\begin{tabular}{lccccc}
\hline Sample & $\mathrm{M}_{w}$ & $\mathrm{M}_{w} \mathrm{M}_{n}$ & $\mathrm{n}_{w}$ & $10^{-4}\left\langle S^{2}\right\rangle\left(\AA^{2}\right)$ & $10^{4} A_{2}\left(\mathrm{~cm}^{3} \mathrm{molg}^{-2}\right)$ \\
\hline PDtBF4 & $4.35 \times 10^{4}$ & 1.14 & 382 & - & 3.31 \\
PDtBF5 & $4.73 \times 10^{4}$ & 1.15 & 415 & - & 3.47 \\
PDtBF8 & $7.98 \times 10^{4}$ & 1.17 & 700 & 2.58 & 2.88 \\
PDtBF9 & $8.88 \times 10^{4}$ & 1.21 & 779 & 3.07 & 3.24 \\
PDtBF15 & $1.46 \times 10^{5}$ & 1.22 & 1280 & 6.15 & 2.43 \\
PDtBF17 & $1.73 \times 10^{5}$ & 1.24 & 1520 & 7.41 & 2.33 \\
\hline
\end{tabular}

Abbreviations: PDtBF, poly(di-tert-butyl fumarate); SLS, static light scattering. 


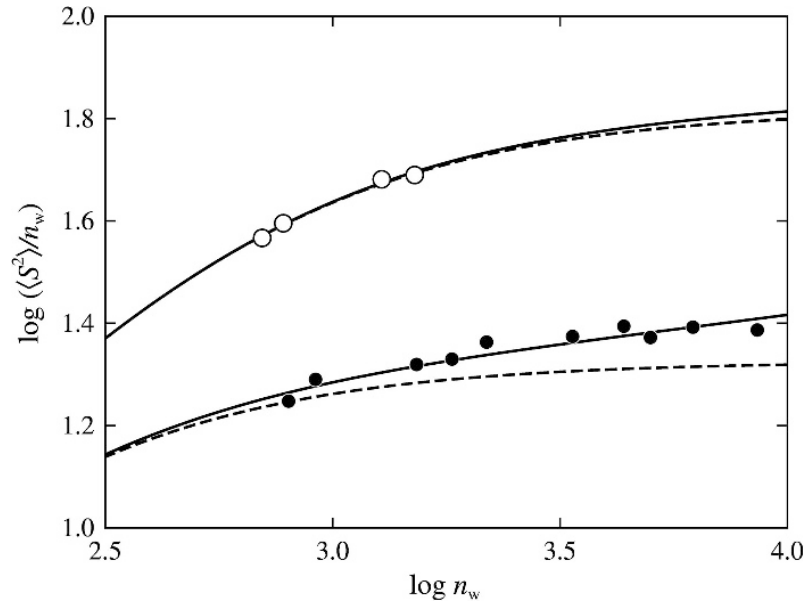

Figure 1 Double-logarithmic plots of $\left\langle S^{2}\right\rangle / n_{\mathrm{w}}\left(\left\langle S^{2}\right\rangle\right.$ in $\left.\AA^{2}\right)$ against $n_{\mathrm{w}}$ : $(0)$ present data for PDtBF in THF at $30.0^{\circ} \mathrm{C}$; $(\mathbf{O})$ previous data for PDiPF in THF at $30.0^{\circ} \mathrm{C} .{ }^{1}$ The solid and dashed curves represent the best-fit theoretical values for the KP chain with and without the excluded volume, respectively.

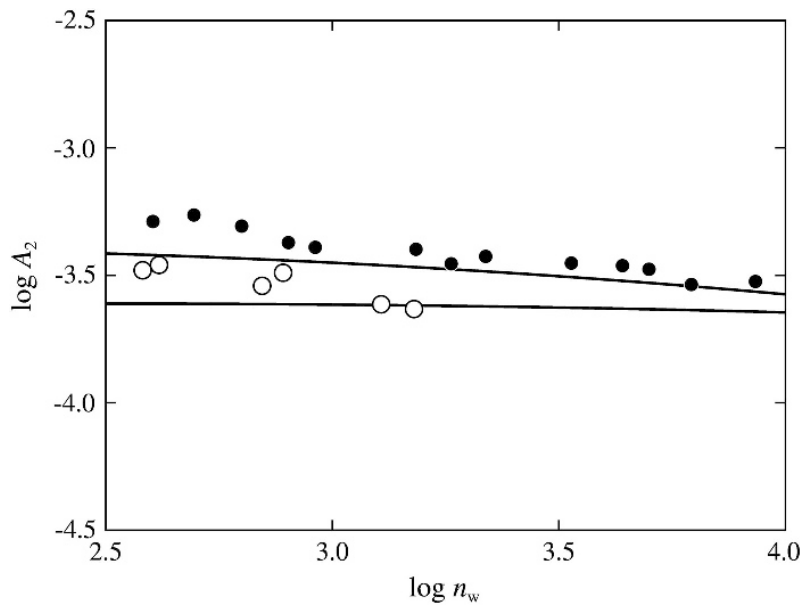

Figure 2 Double-logarithmic plots of $A_{2}$ (in $\mathrm{cm}^{3} \mathrm{~mol} \mathrm{~g}^{-2}$ ) against $n_{\mathrm{w}}$. All of the symbols have the same meaning as those in Figure 1. The solid curves represent the theoretical values for the KP chain without the effects of chain ends (see the text).

For comparison, the previous data for PDiPF in THF at $30.0^{\circ} \mathrm{C}^{1}$ are also plotted in the figure (filled circles). The values of $\left\langle S^{2}\right\rangle / n_{\mathrm{w}}$ for PDtBF are definitely larger than those for PDiPF, indicating that the average dimension of the polymer chains for PDtBF is much larger than that for PDiPF probably because of a remarkable difference in the chain stiffness between the two polymers.

\section{Second virial coefficient $A_{2}$}

The values of $A_{2}$ determined from the SLS measurements in THF at $30.0^{\circ} \mathrm{C}$ for all of the PDtBF samples are listed in the sixth column of Table 1.

Figure 2 shows the double-logarithmic plots of $A_{2}\left(\right.$ in $\mathrm{cm}^{3} \mathrm{~mol} \mathrm{~g}^{-2}$ ) against $n_{\mathrm{w}}$ for PDtBF in THF at $30.0^{\circ} \mathrm{C}$ (unfilled circles). It includes the previous data for PDiPF in THF at $30.0^{\circ} \mathrm{C}^{1}$ (filled circles), for comparison. The solid curves represent the theoretical values for the KP chain without the effects of the chain ends, which are discussed in the Discussion section. The values of $A_{2}$ for PDtBF are smaller than
Table 2 Results of the viscosity and DLS measurements for poly (di-tert-butyl fumarate) in tetrahydrofuran at $30.0^{\circ} \mathrm{C}$

\begin{tabular}{lccccc}
\hline Sample & {$[\eta]\left(d l g^{-1}\right)$} & $\mathrm{k}^{\prime}$ & $10^{7} \mathrm{D}\left(\mathrm{cm}^{2} \mathrm{~s}^{-1}\right)$ & $\mathrm{k}_{\mathrm{D}}{ }^{(L S)}\left(\mathrm{cm}^{3} \mathrm{~g}^{-1}\right)$ & $\mathrm{R}_{H}(\AA)$ \\
\hline PDtBF4 & 0.375 & 0.51 & - & - & - \\
PDtBF5 & 0.378 & 0.51 & 7.09 & 11 & 71 \\
PDtBF8 & 0.670 & 0.50 & 4.89 & 21 & 103 \\
PDtBF9 & 0.775 & 0.38 & 4.23 & 32 & 119 \\
PDtBF15 & 1.07 & 0.47 & 3.36 & 38 & 150 \\
PDtBF17 & 1.27 & 0.47 & 3.01 & 30 & 168 \\
\hline
\end{tabular}

Abbreviations: PDtBF, poly(di-tert-butyl fumarate); DLS, dynamic light scattering.

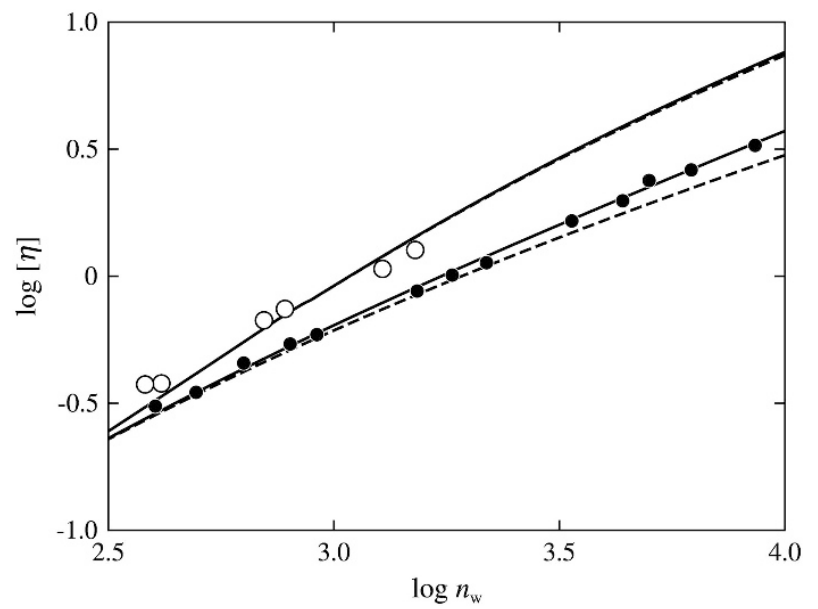

Figure 3 Double-logarithmic plots of $[\eta]$ (in $\mathrm{dl} \mathrm{g}^{-1}$ ) against $n_{\mathrm{w}}$ : (०) present data for PDtBF in THF at $30.0^{\circ} \mathrm{C}$; $(\bullet)$ previous data for PDiPF in THF at $30.0^{\circ} \mathrm{C} .{ }^{2}$ The solid and dashed curves represent the best-fit theoretical values for the KP chain with and without the excluded volume, respectively.

those for PDiPF, implying that THF at $30.0^{\circ} \mathrm{C}$ is not as good a solvent for PDtBF as it is for PDiPF.

Intrinsic viscosity $[\eta]$

The values of $[\eta]$ and $k^{\prime}$ determined from the viscosity measurements in THF at $30.0^{\circ} \mathrm{C}$ for all of the PDtBF samples are listed in the second and third columns of Table 2. The values of $k^{\prime}$ for PDtBF are larger than those for PDiPF $\left(k^{\prime}=0.3-0.4\right)$ previously reported. ${ }^{2}$ This result is consistent with the fact that THF at $30.0^{\circ} \mathrm{C}$ is a better solvent for $\mathrm{PDiPF}$ than it is for PDtBF, as deduced from the above-mentioned result of $A_{2}$.

Figure 3 shows the double-logarithmic plots of $[\eta]$ (in $\mathrm{dl} \mathrm{g}^{-1}$ ) against $n_{\mathrm{w}}$ for $\mathrm{PDtBF}$ in $\mathrm{THF}$ at $30.0^{\circ} \mathrm{C}$ (unfilled circles). For comparison, it includes the previous results for PDiPF in THF at $30.0^{\circ} \mathrm{C}^{2}$ (filled circles). All of the curves have the same meaning as those in Figure 1. As in the case of $\left\langle S^{2}\right\rangle$, the values of $[\eta]$ for PDtBF are appreciably larger than those for PDiPF, indicating that the effective hydrodynamic (molar) volume is much larger for PDtBF than it is for PDiPF, probably because of a large difference in the chain stiffness between the two polymers.

Translational diffusion coefficient $D$ and hydrodynamic radius $R_{\mathrm{H}}$ The values of $D$ and $k_{\mathrm{D}}{ }^{(\mathrm{LS})}$ determined from the DLS measurements in THF at $30.0^{\circ} \mathrm{C}$ for all of the PDtBF samples except PDtBF4 are listed in the fourth and fifth columns of Table 2. We note that $k_{\mathrm{D}}{ }^{(\mathrm{LS})}$ is concerned with the coefficient of the concentration $c$ in the expansion 


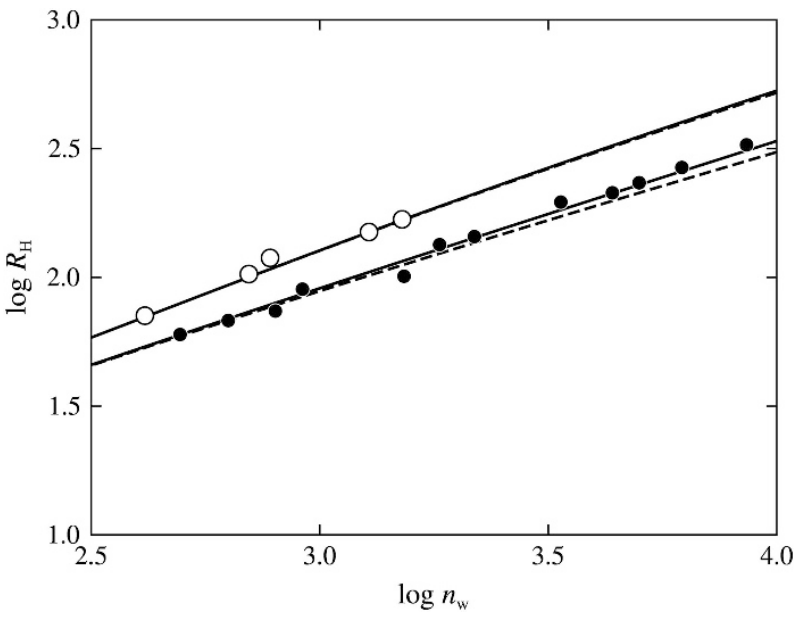

Figure 4 Double-logarithmic plots of $R_{\mathrm{H}}$ (in $\AA$ ) against $n_{\mathrm{w}}$. All of the symbols have the same meaning as those in Figure 3 . The solid and dashed curves represent the best-fit theoretical values for the KP chain with and without the excluded volume, respectively.

of the apparent diffusion coefficient $D^{(\mathrm{LS})}(c)$ at finite $c{ }^{18}$ In the last column of Table 2 are also listed the values of the hydrodynamic radius $R_{\mathrm{H}}$ calculated from the defining equation

$$
R_{\mathrm{H}}=k_{\mathrm{B}} T / 6 \pi \eta_{0} D
$$

where $k_{\mathrm{B}}$ is the Boltzmann constant and $T$ is the absolute temperature.

In Figure $4, R_{\mathrm{H}}$ (in $\AA$ ) is double-logarithmically plotted against $n_{\mathrm{w}}$ for PDtBF in THF at $30.0^{\circ} \mathrm{C}$ (unfilled circles). For comparison, the previous results for PDiPF in THF at $30.0^{\circ} \mathrm{C}^{2}$ are also included in the figure (filled circles). All of the curves have the same meaning as those in Figure 1. As in the cases of $\left\langle S^{2}\right\rangle$ and $[\eta]$, the values of $R_{\mathrm{H}}$ for PDtBF are larger than those for PDiPF.

\section{DISCUSSION}

\section{Analyses of $\left\langle S^{2}\right\rangle$ and $A_{2}$ on the basis of the KP model}

In this section, we simultaneously analyze the present data of $\left\langle S^{2}\right\rangle$ and $A_{2}$ for PDtBF on the basis of the KP model with consideration of both the intramolecular and intermolecular excluded-volume effects and determine the model parameter values for PDtBF. The KP chain ${ }^{6,7}$ itself is an elastic wire model that possesses only a bending energy and may be characterized by the stiffness parameter $\lambda^{-1}$ having the dimension of length. The KP chain model is a special case of the helical wormlike (HW) chain ${ }^{7}$ model, which is a more general polymer chain model having a torsional energy in addition to the bending energy.

For the KP chain of total contour length $L,\left\langle S^{2}\right\rangle$ may be written in the form,

$$
\left\langle S^{2}\right\rangle=\left\langle S^{2}\right\rangle_{0} \alpha_{S}^{2}
$$

where $\left\langle S^{2}\right\rangle_{0}$ is the unperturbed mean-square radius of gyration given as a function of $\lambda^{-1}$ and $L$ by equation 5 of Nakatsuji et al. ${ }^{1,7,22}$ In Equation $3, \alpha_{S}$ is the gyration-radius expansion factor concerned with the intramolecular excluded-volume effect. In the framework of the QTP scheme, ${ }^{7,10-12} \alpha_{S}$ is a function only of the intramolecular scaled excluded-volume parameter $\tilde{z}$, and for the function of $\alpha_{S}$, we usually use the Domb-Barrett equation ${ }^{23}$ given by equation 6 of Nakatsuji et al. ${ }^{1}$ The parameter $\tilde{z}$ is defined as a product of a function $3 / 4 \times$ $K(\lambda L)$ and the conventional excluded-volume parameter $z$ defined in the two-parameter (TP) scheme. ${ }^{7,24}$ The function $K(\lambda L)$ of the reduced contour length $\lambda L$ represents the effects of the chain stiffness a

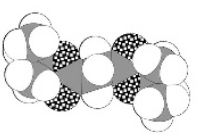

b

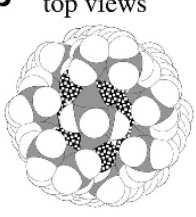

side views
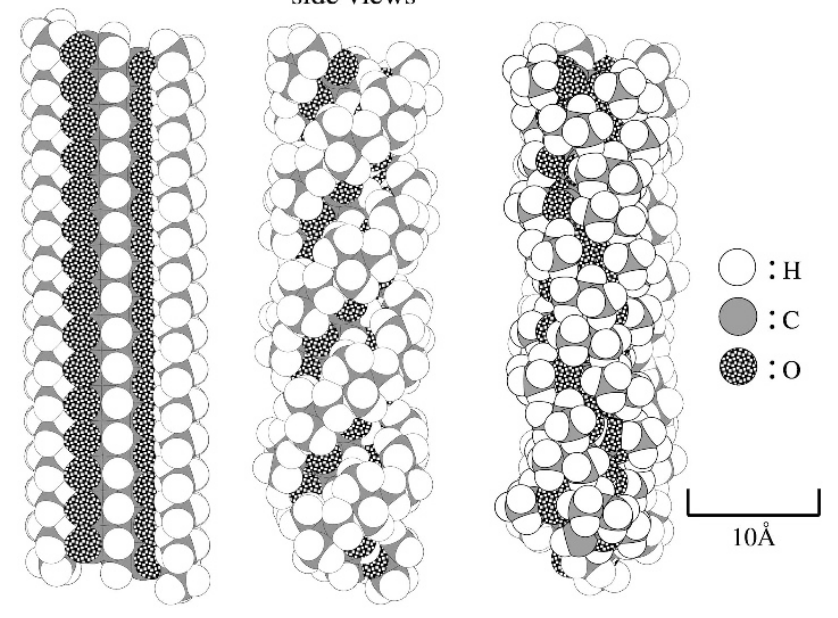

C

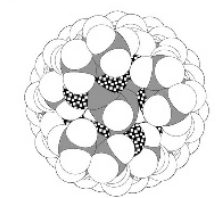

Figure 5 Schematic drawing of the chain conformations of PDtBF with $f_{r}=0$, where all of the atoms are depicted by spheres with diameters corresponding to the van der Walls radii: (a) the planar trans conformation with $\phi=0$; (b) the helical conformation with $\phi=30^{\circ}$; (c) the helical conformation with $\phi=60^{\circ}$.

on the intramolecular excluded-volume effect and is given by equation 8 of Nakatsuji et al. ${ }^{1}$ The parameter $z$ may be written by using the reduced excluded-volume strength $\lambda B$ and $\lambda L$, as obtained with equation 9 of Nakatsuji et al. ${ }^{1}$ The excluded-volume strength $B$ is related to the binary-cluster integral $\beta$ of the beads, which are arrayed along the KP chain in order to incorporate the excluded volume into the chain. ${ }^{7}$

In the theory by Yamakawa et al., ${ }^{7,13} A_{2}$ of the KP chain may then be written in the form,

$$
A_{2}=A_{2}^{(\mathrm{HW})}+A_{2}^{(\mathrm{E})}
$$

where $A_{2}{ }^{(\mathrm{HW})}$ is a part of $A_{2}$ without the effects of the chain ends and $A_{2}{ }^{(E)}$ represents the contribution of the effects of the chain ends. The superscript (HW) attached to the first term indicates that the theory is originally constructed on the basis of the HW chain. For a KP chain of total length $L$ with an excluded-volume strength $B, A_{2}{ }^{(\mathrm{HW})}$ may be given as a function of $\lambda^{-1}, M_{\mathrm{L}}, \lambda B$ and $\lambda L$ by using equation 13 together with equations 6-9 and 14-18 of Nakatsuji et al. ${ }^{1}$ We note that the second term $A_{2}{ }^{(E)}$ in Equation 4 rapidly decreases to 0 with increasing molecular weight $M$ and may be neglected for $M \gtrsim 10^{5}$ for all of the polymer-solvent systems examined so far. ${ }^{7,25-29}$

To compare the above-mentioned KP theoretical values of $\left\langle S^{2}\right\rangle$ and $A_{2}$ calculated as functions of $L$ with the experimental ones as functions of $M$ or the number of repeat units $n$ in the polymer chain, $L$ is related to $M$ or $n$ by

$$
L=M / M_{\mathrm{L}}=n M_{0} / 2 M_{\mathrm{L}}
$$

with the shift factor $M_{\mathrm{L}}$. In this manner, we may in principle determine the three parameters $\lambda^{-1}, M_{\mathrm{L}}$ and $\lambda B$ by simultaneous curve fitting of the KP theoretical values to the experimental data for $\left\langle S^{2}\right\rangle$ and $A_{2}$. 
Unfortunately, however, the range $382-1520$ of $n_{\mathrm{w}}$ for the present PDtBF samples is not sufficiently wide to unambiguously determine all of the KP model parameters from such an analysis. In order to reduce the number of the parameters to be determined, we assume the value of $M_{\mathrm{L}}$ for PDtBF to be $101 \AA^{-1}$. This value has been calculated by assuming that the ratio of the value of $M_{\mathrm{L}}$ for PDtBF to that for PDiPF $\left(89 \AA^{-1}\right)^{1}$ is equal to the ratio of the value of $M_{0}$ for the former (228) to that for the latter (200). To the best of our knowledge, there are no available data for the crystal structure of PDtBF. Therefore, in order to confirm whether the above-assigned value of $101 \AA^{-1}$ for $M_{\mathrm{L}}$ is reasonable or not, we estimate $M_{\mathrm{L}}$ on the basis of the chain conformations of PDtBF. In Figure 5a, the PDtBF chain (with $n=30$ and $f_{\mathrm{r}}=0$ ) in the planar trans conformation (with an internal rotation angle $\phi$ of $0^{\circ}$ and with a bond angle of $109^{\circ}$ ) is schematically illustrated, whose $M_{\mathrm{L}}$ may be estimated as $95 \AA^{-1}$. The $M_{\mathrm{L}}$ value for a polymer chain in a helical conformation generally becomes larger than that for the chain in the planar trans conformation, so the (true) $M_{\mathrm{L}}$ value for the PDtBF chain may be larger than $95 \AA^{-1}$. For the PDtBF chain (with $n=30$ and $f_{\mathrm{r}}=0$ ) in helical conformations, we demonstrate two chains having fixed $\phi=30^{\circ}$ and $60^{\circ}$, which are schematically illustrated in Figures $5 \mathrm{~b}$ and $\mathrm{c}$, respectively. The values of $M_{\mathrm{L}}$ are estimated to be 96 and $100 \AA^{-1}$ for $\phi=30^{\circ}$ and $60^{\circ}$, respectively. These values do not significantly differ from $95 \AA^{-1}$ (and also from $\left.101 \AA^{-1}\right)$. In order to investigate the preferred chain conformation of PDtBF, the PDtBF chains for $\phi=0^{\circ}, 30^{\circ}$ and $60^{\circ}$ were generated with AMBER $12^{30}$ using a generalized amber force field (gaff). Then it was found that the chain conformation of PDtBF with the lowest total conformational energy (in vacuum) is in the $10_{1}$ helical conformation, where the rotation angles associated with two successive main-chain bonds are alternately $0^{\circ}$ and $60^{\circ}$. The value of $M_{\mathrm{L}}$ for this $10_{1}$ helical conformation is evaluated as $95 \AA^{-1}$. Considering these facts, the value $101 \AA^{-1}$ of $M_{\mathrm{L}}$ assigned above is consistent with those estimated on the basis of the chain conformations of PDtBF. Thus we adopt the value $101 \AA^{-1}$ of $M_{\mathrm{L}}$ and then attempt to determine the remaining two model parameters, $\lambda^{-1}$ and $\lambda B$, from a simultaneous comparison of the KP theories of $\left\langle S^{2}\right\rangle$ and $A_{2}$ with the experimental data.

In Figures 1 and 2, the solid curves associated with the present data points for PDtBF represent the best-fit KP theory values of $\left\langle S^{2}\right\rangle$ and those of $A_{2}$ without a consideration of the effects of chain ends, that is, $A_{2}{ }^{(\mathrm{HW})}$, respectively, where these theoretical values have been calculated with $\lambda^{-1}=350 \AA$ and $\lambda B=0.024$ along with the abovementioned value $101 \AA^{-1}$ of $M_{\mathrm{L}}$. We note that the determination errors in the values of $\lambda^{-1}$ and $\lambda B$ for PDtBF are evaluated as $\pm 7 \%$ and $\pm 8 \%$, respectively, at most. In the figures, the solid curves associated with the data points for PDiPF represent the best-fit KP theory values calculated in the same manner as in the case of PDtBF, with the model parameter values previously determined ${ }^{1}$ $\left(\lambda^{-1}=113 \AA, M_{\mathrm{L}}=89 \AA^{-1}\right.$ and $\left.\lambda B=0.097\right)$. It is seen from the figures that for PDtBF the theoretical values may well reproduce the experimental values of $\left\langle S^{2}\right\rangle$ over the whole range of $n_{\mathrm{w}}$ examined and those of $A_{2}$ in the range of $n_{\mathrm{w}} \gtrsim 10^{3}$, where the effects of the chain ends may be negligibly small. The value $350 \AA$ of $\lambda^{-1}$ determined for $\mathrm{PDtBF}$ is appreciably larger than that for PDiPF, as is expected from their chemical structures. Therefore, it may be concluded that the difference in the substituents between PDtBF and PDiPF, that is, ester tert-butyl group and ester isopropyl one, has a great influence on the chain stiffness and also on the average chain dimensions of the two polymers. In Figure 2, the data points for each polymer are seen to deviate progressively upward from the theoretical curve with decreasing $n_{\mathrm{w}}$ for $n_{\mathrm{w}} \lesssim 10^{3}$, because of the effects of the chain ends $\left(A_{2}{ }^{(\mathrm{E})}\right)$.
Concerning the side-chain group dependence of the average chain dimension, it is pertinent to refer to the literature data ${ }^{31-33}$ for poly (methacrylic acid) derivatives (that is, polymethacrylates). The data show that an increase in size of side-chain groups of the polymethacrylates is accompanied by an increase in the coil-limiting value $C_{\infty}$ of the characteristic ratio, that is, an increase in the average chain dimension. This trend is consistent with the results obtained for PDiPF and PDtBF.

In Figure 1, the theoretical values of $\left\langle S^{2}\right\rangle_{0}$ (dashed curves) for the respective polymers, calculated with the same model parameter values of $\lambda^{-1}$ and $M_{\mathrm{L}}$ as those mentioned above, are also shown. It is seen from the figure that the present data points for PDtBF agree with the theoretical values of $\left\langle S^{2}\right\rangle_{0}$, as well as those of $\left\langle S^{2}\right\rangle$ (solid curve), for the whole range of $n_{\mathrm{w}}$ examined. On the other hand, the data points for PDiPF are seen to deviate progressively upward from the theoretical $\left\langle S^{2}\right\rangle_{0}$ with increasing $n_{\mathrm{w}}$. These results indicate that the intramolecular excluded-volume effect may be ignored for PDtBF in the range of $n_{\mathrm{w}}$ examined, while the effect cannot be ignored for PDiPF because the PDiPF chain is much more flexible than the PDtBF one.

\section{Analysis of $[\eta]$ on the basis of the KP model}

Next we proceed to analyze the data for $[\eta]$ on the basis of the unperturbed KP cylinder model ${ }^{7,34,35}$ combined with the QTP theory. ${ }^{7,10-12}$

For the perturbed KP cylinder model with excluded volume, $[\eta]$ may be expressed as

$$
[\eta]=[\eta]_{0} \alpha_{\eta}^{3}
$$

where $[\eta]_{0}$ is the intrinsic viscosity for the unperturbed KP cylinder model of total length $L$ and diameter $d$ and may be given as a function of $\lambda^{-1}, \lambda L$ and the reduced diameter $\lambda d$ by using equation 6.89 with equation 6.90 of Yamakawa and Yoshizaki ${ }^{7}$ for $\lambda L \geqslant 2.278$ and equation 6.91 with equations 6.92 and 6.94 of Yamakawa and Yoshizaki $^{7}$ for $\lambda L<2.278$. In Equation $6, \alpha_{\eta}$ is the viscosity-radius expansion factor. In the framework of the QTP scheme, ${ }^{7,10-12} \alpha_{\eta}$ is a function only of $\tilde{z}$. As usual, ${ }^{7}$ for $\alpha_{\eta}$, we adopt the Barrett equation ${ }^{36}$ given by equation 17 of Nakatsuji et al. ${ }^{2}$

On the basis of the theory, the perturbed KP theory value of [ $\eta$ ] may be calculated as a function of $M$ or $n$ for a given set of values of the four model parameters $\lambda^{-1}, M_{\mathrm{L}}, \lambda B$ and $d$. Namely, we can determine the parameters for PDtBF from curve fitting to the experiment data for $[\eta]$. However, as seen from Figure 3, the increase in $[\eta]$ for PDtBF with increasing $n_{\mathrm{w}}$ is monotonic, and the plot does not show any prominent feature such as an S-shaped curve, which was observed for $\operatorname{poly}\left(n\right.$-hexyl isocyanate) ${ }^{9}$ Therefore, it is difficult to determine all of the parameters from the curve fitting. Thus we assume values of $350 \AA$ and 0.024 for $\lambda^{-1}$ and $\lambda B$, respectively, as determined above from the analyses of $\left\langle S^{2}\right\rangle$ and $A_{2}$ and then determine the two parameters $M_{\mathrm{L}}$ and $d$, as done in the case of PDiPF. $^{2}$

In Figure 3, the solid curve associated with the data points for PDtBF represents the best-fit perturbed KP theory values for PDtBF calculated with $M_{\mathrm{L}}=123 \AA^{-1}$ and $d=17 \AA$ and the above-mentioned values of $\lambda^{-1}$ and $\lambda B$. In the figure, the solid curve associated with the data points for PDiPF represents the best-fit perturbed KP theory values for PDiPF, calculated with the model parameter values previously determined ${ }^{2}$ for $\operatorname{PDiPF}\left(\lambda^{-1}=113 \AA, \quad M_{\mathrm{L}}=92 \AA^{-1}\right.$, $d=15 \AA$ and $\lambda B=0.097)$. The experimental values are in good agreement with those of the perturbed KP theory calculated with the model parameter values, which are consistent with those determined from $\left\langle S^{2}\right\rangle$ and $A_{2}$ for each polymer. A value of $17 \AA$ for $d$ for 
PDtBF is close to the value of $15 \AA$ for PDiPF, indicating that the difference in the substituents between the two polymers has no significant effect on the (hydrodynamic) chain thickness of them but remarkably affects the chain stiffness.

\section{Analysis of $R_{\mathrm{H}}$ on the basis of the KP model}

In this subsection, we analyze the data for $R_{\mathrm{H}}$ on the basis of the unperturbed KP cylinder model ${ }^{7,37,38}$ combined with the QTP theory. ${ }^{7,10-12}$

For the perturbed KP cylinder model with excluded volume, $R_{\mathrm{H}}$ may be expressed as

$$
R_{\mathrm{H}}=R_{\mathrm{H}, 0} \alpha_{\mathrm{H}}
$$

where $R_{\mathrm{H}, 0}$ is the hydrodynamic radius for the unperturbed KP cylinder model of total length $L$ and diameter $d$ and may be given as a function of $\lambda^{-1}, \lambda L$, and $\lambda d$ by using equations $6.41-6.43$ with equations 6.22 and 6.23 of Yamakawa and Yoshizaki. ${ }^{7}$ In Equation 7, $\alpha_{\mathrm{H}}$ is the hydrodynamic-radius expansion factor. In the framework of the QTP scheme, ${ }^{7,10-12} \alpha_{\mathrm{H}}$ is also a function only of $\tilde{z}$ and may be expressed as a product of $\alpha_{\mathrm{H}, 0}$, for which we usually use the Barrett equation $^{39}$ given by equation 24 of Nakatsuji et al., ${ }^{2}$ and $h_{\mathrm{H}}$, which represents a possible effect of the fluctuating hydrodynamic interaction on $\alpha_{\mathrm{H}}$ and is given by equation 25 of Nakatsuji et al. $2,7,40$

The perturbed KP theory of $R_{\mathrm{H}}$ may also be calculated as a function of $M$ or $n$ for a given set of values of the four model parameters $\lambda^{-1}$, $M_{\mathrm{L}}, \lambda B$ and $d$. As in the case of $[\eta]$, we assume the values of $350 \AA$ and 0.024 for $\lambda^{-1}$ and $\lambda B$, respectively, and determine the two parameters $M_{\mathrm{L}}$ and $d$ from curve fitting to the experimental data for $R_{\mathrm{H}}$.

In Figure 4, the solid curve associated with the data points for PDtBF represents the best-fit perturbed KP theory values for PDtBF, as calculated with $M_{\mathrm{L}}=80 \AA^{-1}$ and $d=18 \AA$ and the abovementioned values of $\lambda^{-1}$ and $\lambda B$. In the figure, the solid curve associated with the data points for PDiPF represents the best-fit perturbed KP theory values for PDiPF, as calculated with the model parameter values previously determined ${ }^{2}$ for $\operatorname{PDiPF}\left(\lambda^{-1}=113 \AA\right.$, $M_{\mathrm{L}}=81 \AA^{-1}, d=16 \AA$ and $\left.\lambda B=0.097\right)$. The theoretical values may well explain the behavior of the experimental data points for each polymer. A value of $80 \AA^{-1}$ for $M_{\mathrm{L}}$ determined from $R_{\mathrm{H}}$ for PDtBF is relatively small compared with the $123 \AA^{-1}$ determined from $[\eta]$ and the $101 \AA^{-1}$ used in the analyses of $\left\langle S^{2}\right\rangle$ and $A_{2}$. This difference may be attributed to the discrepancy between the theoretical and experimental values of the coil-limiting value $\Phi_{\infty}$ of the Flory-Fox factor and the coil-limiting value $\rho_{\infty}$ of the ratio of $\left\langle S^{2}\right\rangle_{0}^{1 / 2}$ to $R_{\mathrm{H}, 0}$, as previously mentioned. ${ }^{7,17}$ Taking account of this fact, we arrive at the conclusion that the unperturbed KP theory combined with the QTP theory may comprehensively explain the behavior of the experimental values for the four properties $\left\langle S^{2}\right\rangle, A_{2},[\eta]$ and $R_{\mathrm{H}}$ for PDtBF as well as for PDiPF.

In Figure 4 (also in Figure 3), the dashed curves represent the unperturbed KP theory values calculated with the same model parameter values of $\lambda^{-1}, M_{\mathrm{L}}$ and $d$ as those used in the calculations of the perturbed KP theory ones for the respective polymers. The present data points for PDtBF agree with the unperturbed KP theory values as well as the perturbed theory ones, indicating that the intramolecular excluded-volume effects on $R_{\mathrm{H}}$ (and $[\eta]$ ) of PDtBF may be ignored in the range of $n_{\mathrm{w}}$ examined, as in the case of $\left\langle S^{2}\right\rangle$. As for PDiPF, the experimental values and those of the perturbed KP theory are in good agreement with the unperturbed KP theory values for $n_{\mathrm{w}} \lesssim 10^{3}$ but deviate progressively upward from the latter with increasing $n_{\mathrm{w}}$ for $n_{\mathrm{w}} \gtrsim 10^{3}$ because of the intramolecular excludedvolume effect, as previously mentioned. ${ }^{2}$

\section{CONCLUSION}

We have determined $\left\langle S^{2}\right\rangle, A_{2},[\eta]$ and $D$ (and $R_{\mathrm{H}}$ ) for PDtBF in THF at $30.0^{\circ} \mathrm{C}$ in the range of $M_{\mathrm{W}}$ from $4.35 \times 10^{4}$ to $1.73 \times 10^{5}$. From the simultaneous analysis of $\left\langle S^{2}\right\rangle$ and $A_{2}$ on the basis of the corresponding KP theories with a consideration of both the intramolecular and intermolecular excluded-volume effects, $\lambda^{-1}$ and $\lambda B$ for PDtBF have been determined, with $M_{\mathrm{L}}$ having been estimated by analogy with that for PDiPF. The value $350 \AA$ of $\lambda^{-1}$ determined for PDtBF is substantially larger than the value $113 \AA$ for the PDiPF that was previously ${ }^{1}$ studied, indicating that the difference in the substituents between the two polymers causes the remarkable difference in chain stiffness. It has been found that the PDtBF chain is so stiff that the intramolecular excluded-volume effect for $\left\langle S^{2}\right\rangle$ may be ignored in the range of $M_{\mathrm{W}}$ examined. As for $[\eta]$ and $R_{\mathrm{H}}$, the data have been analyzed on the basis of the corresponding theories for the KP cylinder model combined with the QTP theory. It has been shown that the experimental values agree well with the KP theory values using the model parameter values consistent with those determined from $\left\langle S^{2}\right\rangle$ and $A_{2}$. It has also been shown that the intramolecular excludedvolume effects of $[\eta]$ and $R_{\mathrm{H}}$ for PDtBF may be ignored as in the case of $\left\langle S^{2}\right\rangle$.

\section{CONFLICT OF INTEREST}

The authors declare no conflict of interest.

\section{ACKNOWLEDGEMENTS}

This work was supported by JSPS KAKENHI Grant Number 22750111.

1 Nakatsuji, M., Hyakutake, M., Osa, M. \& Yoshizaki, T. Mean-square radius of gyration and second virial coefficient of poly(diisopropyl fumarate) in dilute solution. Polym. J. 40, 566-571 (2008).

2 Nakatsuji, M., Soutoku, K., Osa, M. \& Yoshizaki, T. Transport coefficients of poly (diisopropyl fumarate) in dilute solution. Polym. J. 41, 83-89 (2009).

3 Otsu, T., Minai, H., Toyoda, N. \& Yasuhara, T. Radical high polymerization of dialkyl fumarates with bulky substituents leading to less-flexible rod-like polymers. Makromol. Chem. Suppl. 12, 133-142 (1985).

4 Matsumoto, A., Tarui, T. \& Otsu, T. Dilute solution properties of semiflexible poly (substituted methylenes): intrinsic viscosity of poly(diisopropyl fumarate) in benzene. Macromolecules 23, 5102-5105 (1990).

5 Matsumoto, A. \& Nakagawa, E. Evaluation of chain rigidity of poly(diisopropyl fumarate) from light scattering and viscosity in tetrahydrofuran. Euro. Polym. J. 35, 2107-2113 (1999).

6 Kratky, O. \& Porod, G. Röntgenuntersuchung gelöster fadenmoleküle. Recl. Trav. Chim. Pays Bas 68, 1106-1122 (1949).

7 Yamakawa, H. \& Yoshizaki, T. Helical Wormlike Chains in Polymer Solutions, 2nd edn. (Springer, Berlin, Germany, 2016).

8 Abe, F., Einaga, Y., Yoshizaki, T. \& Yamakawa, H. Excluded-volume effects on the mean- square radius of gyration of oligo- and polystyrenes in dilute solutions. Macromolecules 26, 1884-1890 (1993).

9 Murakami, H., Norisuye, T. \& Fujita, H. Dimensional and hydrodynamic properties of poly(hexyl isocyanate) in hexane. Macromolecules 13, 345-352 (1980).

10 Yamakawa, H. \& Stockmayer, W. H. Statistical mechanics of wormlike chains. II. Excluded volume effects. J. Chem. Phys. 57, 2843-2854 (1972).

11 Yamakawa, H. \& Shimada, J. Stiffness and excluded-volume effects in polymer chains. J. Chem. Phys. 83, 2607-2611 (1985).

12 Shimada, J. \& Yamakawa, H. Statistical mechanics of helical worm-like chains. XV. Excluded-volume effects. J. Chem. Phys. 85, 591-600 (1986).

13 Yamakawa, H. On the theory of the second virial coefficient for polymer chains. Macromolecules 25, 1912-1916 (1992).

14 Otsu, T., Yasuhara, T., Shiraishi, K. \& Mori, S. Radical high polymerization of di-tertbutyl fumarate and novel synthesis of high molecular weight poly(fumaric acid) from its polymer. Polym. Bull. 12, 449 (1984).

15 Crivello, J. V. \& Shim, S.-Y. Deep UV photoresists based on poly(di-tert-butyl fumarate). J. Polym. Sci. Part A Polym. Chem. 33, 513-523 (1995).

16 Berry, G. C. Thermodynamic and conformational properties of polystyrene. I. Light-scattering studies on dilute solutions of linear polystyrenes. J. Chem. Phys., 44, 4550-4564 (1966)

17 Konishi, T., Yoshizaki, T. \& Yamakawa, H. On the "universal constants" $\rho$ and $\Phi$ of flexible polymers. Macromolecules 24, 5614-5622 (1991). 
18 Yamada, T., Yoshizaki, T. \& Yamakawa, H. Transport coefficients of helical wormlike chains. 5. Translational diffusion coefficient of the touched-bead model and its application to oligo- and polystyrenes. Macromolecules 25, 377-383 (1992).

19 Yoshioka, M., Matsumoto, A. \& Otsu, T. meso and racemo additions in propagation for radical polymerization of dialkyl fumarates I. Stereoregularity of poly(dialkyl fumarate)s. Polym. J. 23, 1191-1196 (1991).

20 Yoshioka, M., Matsumoto, A. \& Otsu, T. meso and racemo additions in propagation for radical polymerization of dialkyl fumarates II. Determination of the absolute rate constants. Polym. J. 23, 1249-1252 (1991).

21 Wang, X., Komoto, T., Ando, I. \& Otsu, T. Stereochemical configuration of polyfumarates as studied by ${ }^{13} \mathrm{C}$ nuclear magnetic resonance spectroscopy. Makromol. Chem. 189, 1845-1854 (1988)

22 Benoit, H. \& Doty, P. Light scattering from non-gaussian chains. J. Phys. Chem. 57 958-963 (1953).

23 Domb, C. \& Barrett, A. J. Universality approach to the expansion factor of a polymer chain. Polymer 17, 179-184 (1976).

24 Yamakawa, H. Modern Theory of Polymer Solutions (Harper \& Row, New York, USA 1971). Its electronic edition is available online at the URL http://www.molsci.polym. kyoto-u.ac.jp/archives/redbook.pdf.

25 Kasabo, F., Kanematsu, T., Nakagawa, T., Sato, T. \& Teramoto, A. Solution properties of cellulose tris(phenyl carbamate). 1. Characterization of the conformation and intermolecular interaction. Macromolecules 33, 2748-2756 (2000).

26 Einaga, Y., Abe, F. \& Yamakawa, H. Second virial coefficients of oligo- and polystyrenes. Effects of chain ends. Macromolecules 26, 6243-6250 (1993).

27 Abe, F., Einaga, Y. \& Yamakawa, H. Second virial coefficient of oligo- and poly(methyl methacrylate)s. Effects of chain stiffness and chain ends. Macromolecules 27, 3262-3271 (1994).

28 Kamijo, M., Abe, F., Einaga, Y. \& Yamakawa, H. Second virial coefficient of isotactic oligo- and poly(methyl methacrylate)s. Effects of chain stiffness and chain ends. Macromolecules 28, 4159-4166 (1995).
29 Tokuhara, W., Osa, M., Yoshizaki, T. \& Yamakawa, H. Second virial coefficient of oligoand poly $(\alpha$-methylstyrene)s. Effects of chain stiffness, chain ends, and three-segment interactions. Macromolecules 36, 5311-5320 (2003).

30 Case, D. A., Darden, T. A., Cheatham, T. E. III, Simmerling, C. L., Wang, J., Duke, R. E., Luo, R., Walker, R. C., Zhang, W., Merz, K. M., Roberts, B., Hayik, S., Roitberg, A., Seabra, G., Swails, J., Götz, A. W., Kolossváry, I., Wong, K. F., Paesani, F., Vanicek, J., Wolf, R. M., Liu, J., Wu, X., Brozell, S. R., Steinbrecher, T., Gohlke, H., Cai, Q. Ye, X., Wang, J., Hsieh, M.-J., Cui, G., Roe, D. R., Mathews, D. H., Seetin, M. G., Salomon-Ferrer, R., Sagui, C., Babin, V., Luchko, T., Gusarov, S., Kovalenko, A. \& Kollman, P. A. (2012) AMBER 12, University of California, San Francisco.

31 Zhongde, X., Hadjichristidis, N. \& Fetters, L. Solution properties and chain dimensions of poly(n-alkyl methacrylates). Macromolecules 17, 2303-2306 (1984).

32 Siakali-Kioulafa, E., Hadjichristidis, N. \& Mays, J. Synthesis and solution properties of polymethacrylates with alicyclic side groups. Macromolecules 22, 2059-2062 (1989).

33 Karandinos, A., Nan, S., Mays, J. \& Hadjichristidis, N. Solution properties and unperturbed dimensions of stereoirregular poly(tert-butyl methacrylates). Macromolecules 24, 2007-2010 (1991).

34 Yamakawa, H. \& Fujii, M. Intrinsic viscosity of wormlike chains. Determination of the shift factor. Macromolecules 7, 128-135 (1974).

35 Yamakawa, H. \& Yoshizaki, T. Transport coefficients of helical wormlike chains. 3. Intrinsic viscosity. Macromolecules 13, 633-643 (1980).

36 Barrett, A. J. Intrinsic viscosity and friction coefficients for an excluded volume polymer in the Kirkwood approximations. Macromolecules 17, 1566-1572 (1984).

37 Yamakawa, H. \& Fujii, M. Translational friction coefficient of wormlike chains. Macromolecules 6, 407-415 (1973).

38 Yamakawa, H. \& Yoshizaki, T. Transport coefficients of helical wormlike chains. 3. Translational friction coefficient. Macromolecules 12, 32-38 (1979).

39 Barrett, A. J. Investigation of moments of intrachain distances in linear polymers Macromolecules 17, 1561-1566 (1984)

40 Yamakawa, H. \& Yoshizaki, T. Effects of fluctuating hydrodynamic interaction on the hydrodynamic-radius expansion factor of polymer chains. Macromolecules 28, 3604-3608 (1995). 